\title{
Genomic non-coding regions reveal hidden patterns of mumps virus circulation in Spain, 2005 to 2015
}

Ana M Gavilán ${ }^{1}$, Aurora Fernández-García ${ }^{1,3}$, Angel Rueda1, Ana Castellanos ${ }^{1,3}$, Josefa Masa-Calles ${ }^{2,3}$, Noemí López-Perea ${ }^{2,3}$, María V Torres de Mier ${ }^{2,3}$, Fernando de Ory ${ }^{1,3}$, Juan E Echevarría ${ }^{1,3}$

1. Centro Nacional de Microbiología, Instituto de Salud Carlos III, Majadahonda, Madrid, Spain

2. Centro Nacional de Epidemiología, Instituto de Salud Carlos III, Madrid, Spain

3. CIBER de Epidemiología y Salud Pública (CIBERESP), Madrid, Spain

Correspondence: Aurora Fernández-García (aurorafg@externos.isciii.es)

Citation style for this article:

Gavilán Ana M, Fernández-García Aurora, Rueda Angel, Castellanos Ana, Masa-Calles Josefa, López-Perea Noemí, Torres de Mier María V, de Ory Fernando,

Echevarría Juan E. Genomic non-coding regions reveal hidden patterns of mumps virus circulation in Spain, 2005 to 2015. Euro Surveill. 2018;23(15):pii=17-00349. https://doi.org/10.2807/1560-7917.ES.2018.23.15.17-00349

Background: Since mumps vaccination was introduced in 1981 in Spain, the incidence of the disease has dropped significantly. However, cyclic epidemic waves and outbreaks still occur, despite high vaccination coverage. The World Health Organization (WHO) recommends genotyping to trace the pattern of mumps virus (MuV) circulation. Genotype $\mathrm{H}$ was predominant in Spain, but was replaced in 2005 by genotype $\mathrm{G}$ which has subsequently remained dominant. Of the small hydrophobic protein gene sequences, $78 \%$ are identical and belong to the MuVi/ Sheffield. GBR.1.05/[G]-variant. Aim: Our study aimed to investigate whether the circulation of MuV strains in Spain was continuous after the emergence of genotype $\mathrm{G}$ in 2005. Method: We obtained 46 samples from Spanish patients infected with MuVi/Sheffield.GBR.1.05/[G] during two epidemic waves and analysed them using new molecular markers based on genomic non-coding regions (NCRs) that discriminate subvariants of this virus strain. Results: Phylogenetic analyses of the nucleoprotein-phosphoprotein and matrix protein-fusion protein NCR indicated strain replacement after a drop in incidence in 2009, which had not been detectable by SH sequencing. Clustering of sequences from patients epidemiologically linked in the same outbreak suggests a potential use for these NCRs in outbreak characterisation. Conclusion: We suggest to consider their use in conjunction with the $\mathrm{SH}$ gene in the future WHO recommendations for MuV epidemiological surveillance.

\section{Introduction}

Mumps is a disease caused by the mumps virus (MuV), which belongs to the genus Rubulavirus (family Paramy xoviridae). It is an enveloped virus with a 15,384 nt nonsegmented, negative-sense RNA genome, with seven transcription units that encode: the nucleoprotein $(N)$, the phosphoprotein $(P)$, the matrix protein $(M)$, the fusion protein $(F)$, the small hydrophobic protein $(\mathrm{SH})$, the haemagglutinin-neuraminidase $(\mathrm{HN})$ and the polymerase (L) [1]. Non-coding regions (NCRs) include the long-terminal repeats (LTRS) at the $3^{\prime}$ - and $5^{\prime}$-genomic ends, as well as untranslated regions (UTRs) flanking each coding sequence (CDS) and several intergenic regions (IGs).

MuV is transmitted by direct contact, droplet spread and contaminated fomites. The incubation period is 15-24 days (median: 19 days) [2] and the main clinical manifestations of mumps are fever and swelling of the parotid glands, which occur in $90 \%$ of clinical cases. However, the infection is sub-clinical in up to $30 \%$ of cases, especially in adults. Orchitis is the most frequent complication in post-pubertal males (20-38\%), followed by aseptic meningitis (ca 15\%). Other, less frequent complications include oophoritis (0.5-7\% of female cases), transient deafness (ca $4 \%$ ) and encephalitis (ca $0.1 \%$ ) [3].

Mumps vaccination was introduced into the Spanish childhood immunisation schedule in 1981 as part of the measles, mumps, rubella (MMR) vaccine. The first dose is currently administered at the age of 12 months and the second at 3-4 years [4]. As a result of the sustained increase in vaccination coverage, the incidence dropped from 211 per 100,000 inhabitants in 1982 to 35 per 100,000 inhabitants in 1991 [5]. Although vaccine coverage has exceeded $90 \%$ for both doses since 2003 , the incidence of the disease has remained between 3 and 31 cases per 100,000 inhabitants since 1997 [5]. The virus still generates outbreaks in Spain and causes cyclic epidemic waves which peak every 4-7 years, most recently in 1996, 2000, 2007 and 2013. This situation is similar in other countries where outbreaks also occur despite high vaccination coverage $[6,7]$. In fact, many cases occur in vaccinated people $[8,9,10]$. The cause of this reduced vaccine efficiency remains unclear, but waning immunity [11], incomplete 


\section{FIGURE 1}

Phylogenetic tree of mumps virus SH haplotypes of genotype G

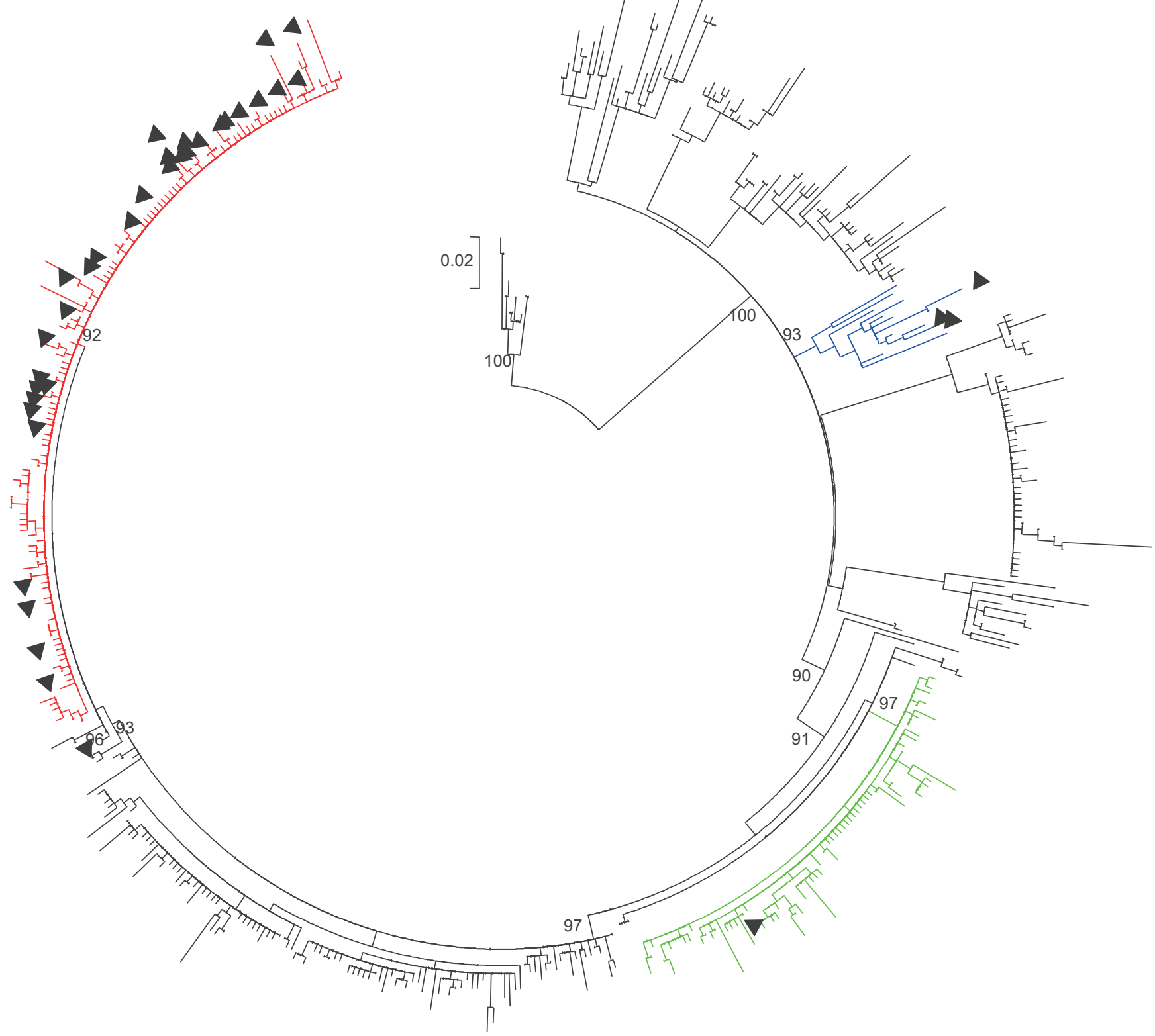

The tree was created with the maximum likelihood method in PhyML, using one sequence of each haplotype of all the sequences from GenBank (until June 2016) and Spanish sequences (January 2005 to June 2016). The GTR+G model of evolution was used and the aLRT SHlike value was analysed. Spanish sequences are indicated with a black triangle. Only support values of aLRT SH-like >9o are shown. Clades including Spanish sequences are shown in colour. The clade comprising the MuVi/Sheffield.GBR/1.05 [G] variant and its related haplotypes is shown in red.

genotype cross-reactivity [12] and antigenic drift [13] have been proposed as explanations.

The World Health Organization (WHO) recommends epidemiological surveillance as a part of the strategy for mumps control [3]. Molecular characterisation of the circulating strains enables identification of circulation patterns and may contribute to the identification of the source of the outbreaks and tracing the transmission chains. Consequently, the WHO has standardised a system for MuV genotyping and a nomenclature for naming strains. Genotyping is based on the sequencing of the $\mathrm{SH}$ and $\mathrm{HN}$ genes. A total of 12 genotypes have been identified: $A, B, C$ (including the former $E$ ), $D, F, G, H, I, J, K$ (including the former $M$ ), L and $N$ [3].

Genotype $\mathrm{K}$ has been reported in the Basque Country (northern Spain) in historical samples collected during 


\section{FIGURE 2}

Phylogenetic trees of mumps virus sequences containing non-coding regions, Spain, 2005-2015 $(\mathrm{n}=46)$

A: N-P NCR

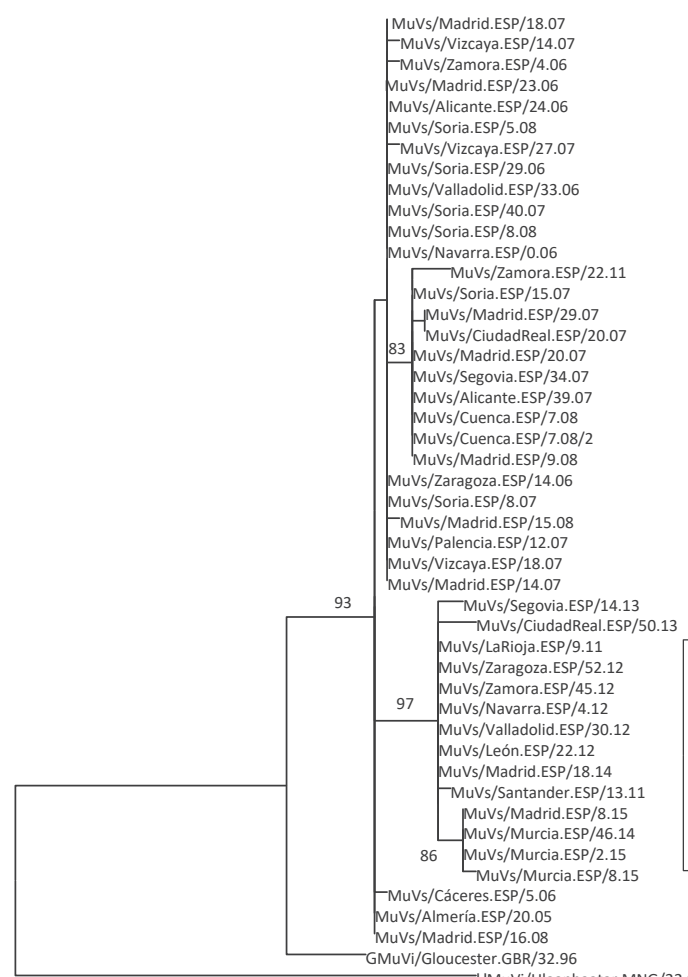

GMuVi/Gloucester.GBR/32.96
B: M-F NCR

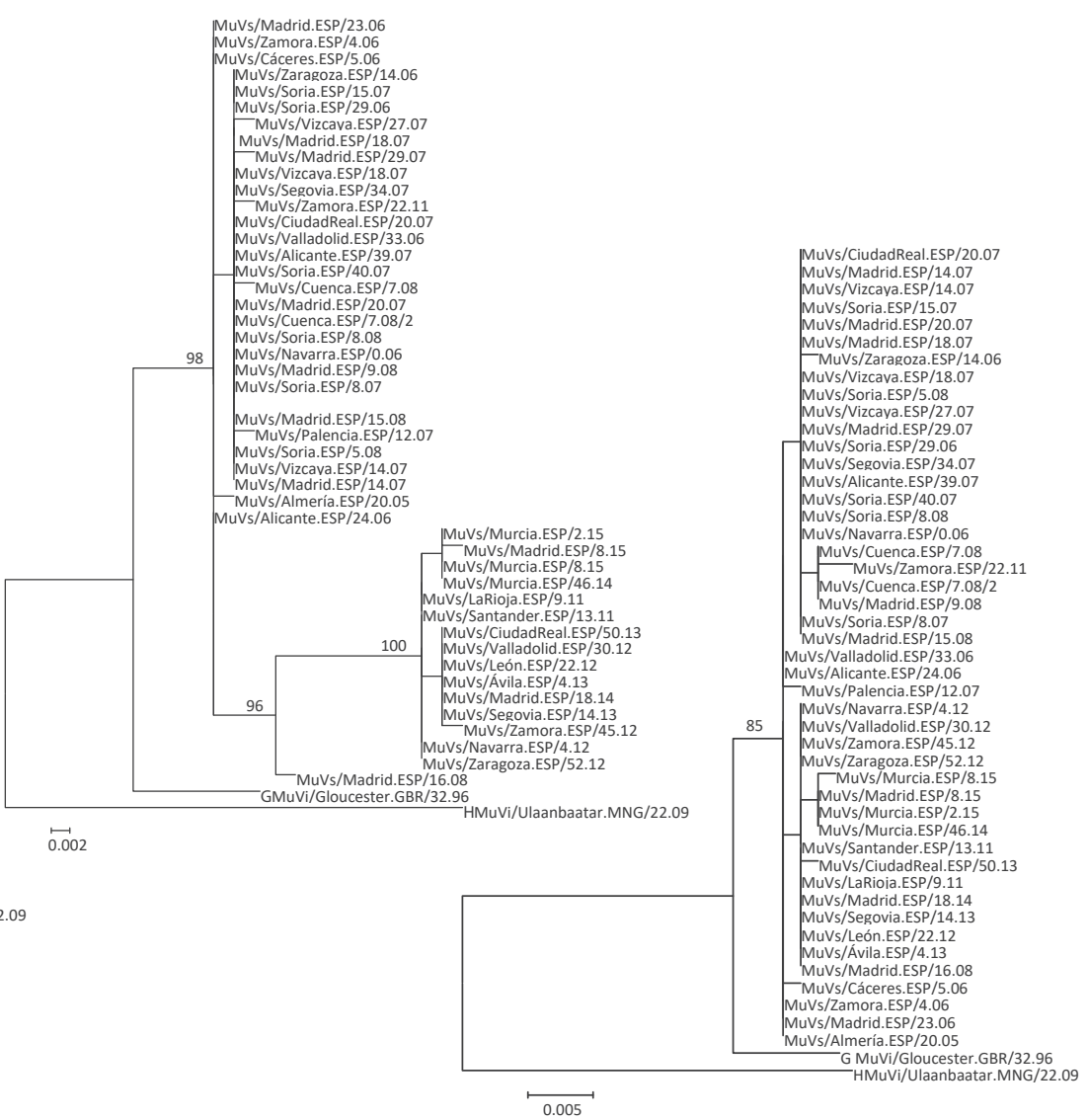

C: P-M NCR

HKY: Hasegawa, Kishino, Yano; MuV: mumps virus.

Analysis was carried out using the maximum likelihood method in PhyML and HKY as the evolutionary model. Statistical analysis involved the use of a bootstrapping approach. The MuV genotype G reference sequence was included (MuVi / Gloucester.GBR / 32.96 [G]), and MuVi / Ulaanbaatar.MNG / $22.09[\mathrm{H}]$ was used as the outgroup.

the years 1987 to 1990 [14]. Genotype H was predominant in Spain from 1996 to 2003, but was replaced in 2005 by genotype $G$ which caused the epidemic wave that peaked in 2007 [15] and also subsequent waves [16]. Other genotypes such as A, C, D and J were detected in sporadic cases or in small, limited outbreaks [15], but genotype $\mathrm{G}$ has remained predominant from 2005 to the present, with no apparent strain replacement between the last two epidemic waves.

The objective of this work was to investigate whether the circulation of MuV strains in Spain was continuous after the emergence of genotype $G$ in 2005 . We evaluated new molecular markers based on the sequencing of NCRs of the MuV genome with the aim of improving the discrimination of genomic variants within genotype G.

\section{Methods}

\section{Samples and RNA extraction}

Among 357 samples from Spanish mumps patients previously characterised as genotype $G$ at the National Center of Microbiology (CNM) according to WHO protocols [16], 78\% were MuVi/Sheffield.GBR.1.05/G variant of the MuV genotype G. For this study, we selected 46 of the MuVi/Sheffield.GBR.1.05/G samples that were representative of all years of the study and different geographical origins. Forty-one of them were saliva samples, two were urine samples, two were nasopharyngeal exudates and one was a viral isolate. Thirty samples were collected during the 2005-2009 epidemic period, and 16 were collected during the 2010-2015 wave. 


\section{FIGURE 3}

Phylogenetic tree of N-P and M-F concatenated sequences, Spain, 2005-2015 ( $\mathrm{n}=45)$

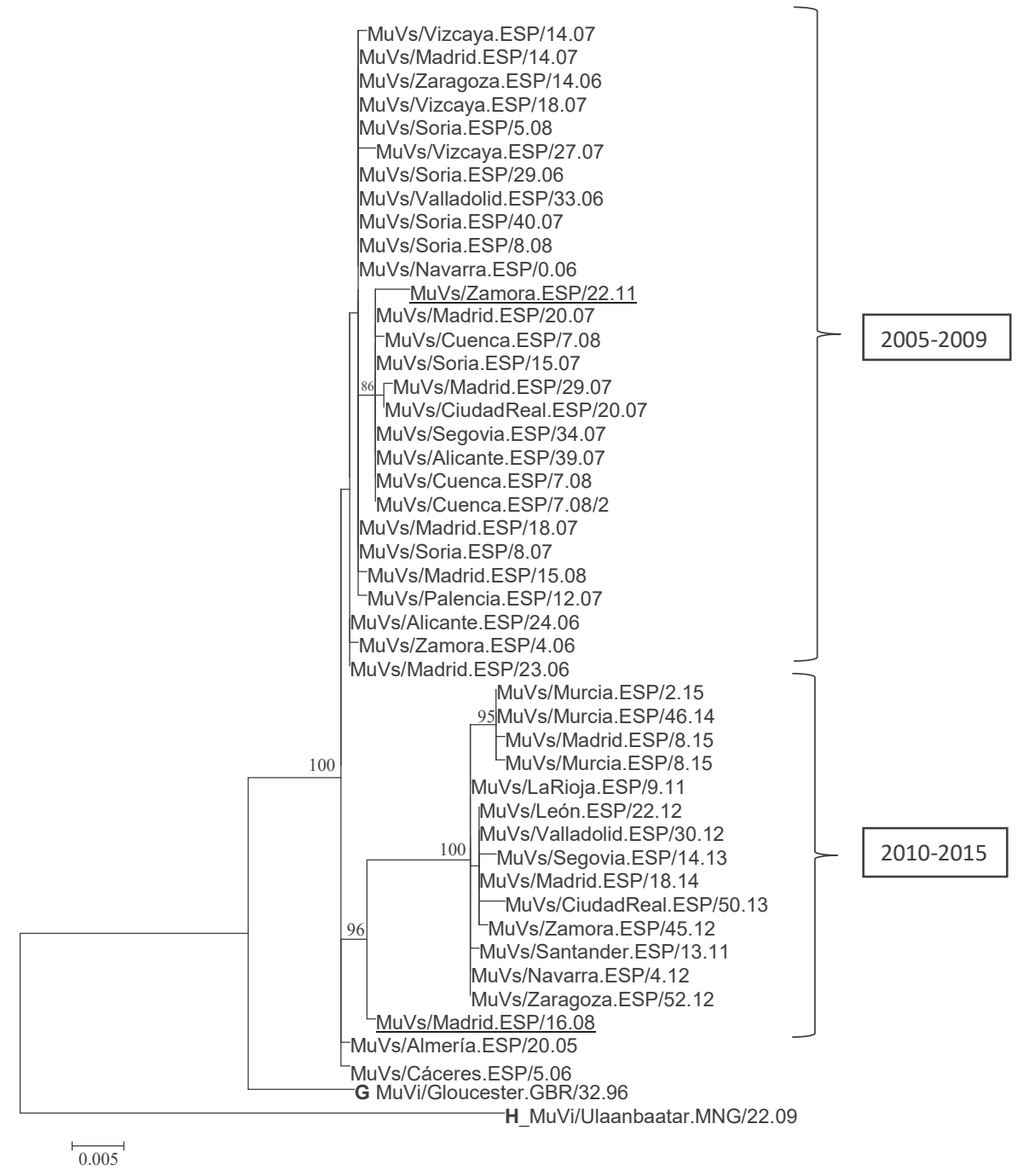

HKY: Hasegawa, Kishino, Yano; MuV: mumps virus.

The tree was derived with the maximum likelihood method in PhyML, using the HKY as the evolutionary model, and a bootstrapping approach for the statistical analysis. The MuV genotype G reference sequence was included (MuVi / Gloucester.GBR / 32.96 [G]) and MuVi / Ulaanbaatar.MNG / $22.09[\mathrm{H}]$ was used as the outgroup. Bootstrap values $>70$ are shown. The two sequences that clustered with sequences from a different time period are underlined.

Nucleic acid was extracted with an automatic extractor (QIAsymphony, QIAGEN) using a commercial kit (QIAsymphony Virus/Bacteria Midi Kit (96); Qiagen). $400 \mu \mathrm{L}$ of sample was extracted to obtain $40 \mu \mathrm{L}$ of final eluate.

\section{Selection of genomic regions, primer design} and amplification conditions

Previous studies of MuV genome variability have shown the NCR located between the N and P CDS (N-P), the $P$ and $M$ CDS (P-M) and the $M$ and F CDS (M-F) to be the most variable, together with the $\mathrm{SH}$ gene recommended by the WHO for genotyping [17]. Fifty-five complete genomes of eight different MuV genotypes (A, B,
C, G, H, I, K, N) were taken from the GenBank database and aligned with MAFFT v.7 [18]. Consensus primers flanking the NCR were designed from the alignment (Table) to amplify fragments containing these regions by nested RT-PCR.

The OneStep RT-PCR kit (QIAGEN, Hilden, Germany) was used for reverse transcription and primary amplification, while nested PCR was performed using the BioTAQ DNA polymerase (Bioline, London, United Kingdom). The reaction mixture contained $0.6 \mu \mathrm{M}$ (N-P and $\mathrm{P}-\mathrm{M})$ or $1 \mu \mathrm{M}(\mathrm{M}-\mathrm{F})$ of each primer (forward and reverse) and $400 \mu \mathrm{M}(\mathrm{N}-\mathrm{P}$ and P-M) or $600 \mu \mathrm{M}(\mathrm{M}-\mathrm{F})$ of dNTPs and $2.5 \mathrm{mM}$ of $\mathrm{MgCl}_{2}$. The thermal profile was $50^{\circ} \mathrm{C}$ for 30 


\begin{tabular}{|c|c|c|c|c|}
\hline Primer name ${ }^{a}$ & Sequence $\left(5^{\prime}-3^{\prime}\right)$ & $\begin{array}{l}\text { Location } \\
\left(\text { nt } 5^{\prime}-3^{\prime}\right)^{\mathrm{b}}\end{array}$ & $\begin{array}{c}\text { Annealing } \\
\text { temperature }\left({ }^{\circ} \mathrm{C}\right)\end{array}$ & Fragment lengthc \\
\hline NP_F1 & САTGCCAYTATATCCTCAAGTCAG & $1,591-1,614$ & \multirow{2}{*}{57} & \multirow{2}{*}{923} \\
\hline NP_R1b & GCATATrGGGTTGCACCACT & $2,557-2,538$ & & \\
\hline NP_F2 & GATTTATTACGATACAACGArAAyGG & $1,673-1,698$ & \multirow{2}{*}{55} & \multirow{2}{*}{774} \\
\hline NP_R2 & TCTATGCCCTCCTCTTGGA & $2,491-2,473$ & & \\
\hline PM_F1 & GCTACAATTGAAGGAATGATGGC & $2,766-2,788$ & \multirow{2}{*}{60} & \multirow{2}{*}{859} \\
\hline PM_R1 & TTGGGGATGCGGTTGATCT & $3,667-3,649$ & & \\
\hline PM_F2 & GTCCCAGTTGATGAGCTTAGAAG & $2,826-2,848$ & \multirow{2}{*}{55} & \multirow{2}{*}{759} \\
\hline PM_R2 & ACTyGCTGTCTTCCGAACC & $3,626-3,608$ & & \\
\hline MF_F1 & TCATCACCATCGTTrGCGAA & $4,185-4,204$ & \multirow{2}{*}{55} & \multirow{2}{*}{504} \\
\hline MF_R1 & GTTATCAGTGGGTTGGATATTyGG & $4,731-4,708$ & & \\
\hline MF_F2 & TGGTCATCTGGGTGTGAAATC & $4,212-4,232$ & \multirow{2}{*}{55} & \multirow{2}{*}{428} \\
\hline MF_R2 & GAGCTTGAACTTTGTGAGTAATAGC & $4,685-4,661$ & & \\
\hline
\end{tabular}

nt: nucleotide.

${ }^{a}$ NP, PM, MF: name of fragment containing the corresponding NCR; F: forward; R: reverse; 1 : primary reaction; 2 : nested reaction.

${ }^{b} \mathrm{MuVi} / \mathrm{Gloucester.GBR/32.96/(AF280799)}$ was used as reference sequence.

' Length excluding primer sequences is presented here.

$\min , 95^{\circ} \mathrm{C}$ for $15 \mathrm{~min}$, then 40 cycles of $94^{\circ} \mathrm{C}$ for $30 \mathrm{~s}$, annealing temperature (Table) for $60 \mathrm{~s}, 72^{\circ} \mathrm{C}$ for $90 \mathrm{~s}$ (N-P and P-M) or $60 \mathrm{~s}(\mathrm{M}-\mathrm{F})$, followed by a final extension step at $72{ }^{\circ} \mathrm{C}$ for $10 \mathrm{~min}$. The nested reaction mixture contained $0.4 \mu \mathrm{M}$ of each primer, $200 \mu \mathrm{M}$ of dNTPs and $2 \mathrm{mM}$ of $\mathrm{MgCl}_{2}$. The thermal profile was $94^{\circ} \mathrm{C}$ for 2 min, then 30 cycles of $94^{\circ} \mathrm{C}$ for $15 \mathrm{~S}, 55^{\circ} \mathrm{C}$ for $30 \mathrm{~s}$ and $72^{\circ} \mathrm{C}$ for $80 \mathrm{~S}$ (N-P and P-M) or $60 \mathrm{~S}(\mathrm{M}-\mathrm{F})$, followed by a final extension step at $72^{\circ} \mathrm{C}$ for $7 \mathrm{~min}$.

\section{Sequencing and phylogenetic analysis}

PCR products were purified by enzymatic reaction Illustra ExoProStar 1-Step (GE Health Care Life Science, Freiburg, Germany) and sequenced with the ABI Big Dye Terminator Cycle Sequencing Kit (Applied Biosystems, Branchburg, NJ, United States) using the corresponding forward and reverse primers. Sequences were edited using BioEdit v.7.2.5 [19] and aligned with MAFFT v.7 [18]. Haplotypes (a set of identical sequences) were identified using DNAsp v5 software [20]. Phylogenetic analysis was performed by maximum likelihood method with the RaxML programme [21] through the BlackBox website (http://phylobench.vital-it.ch/raxml-bb). To confirm the results, the PhyML 3.0 programme [22] was used through the ATGC portal (http://atgc.lirmm.fr/ phyml/). The best evolutionary model was previously selected using jModelTest v.2.1.10 [23] according to the Akaike information criterion. Sequence fragments of the various genes were concatenated using Seaview v.4 [24] and in this case PartitionFinder v. 1.1.1 [25] was used to select the evolutionary model. Phylogenetic trees were edited using MEGA v.6.06 software [26].

\section{GenBank accession numbers}

The sequences obtained in this study have been deposited in GenBank under accession numbers KY607678-KY607722 for N-P NCR, KY607632-KY607677 for P-M NCR, and KY607586 - KY607631 for M-F NCR.

\section{Results}

Analysis of sequence variants

A total of 1,292 samples positive for MuV RNA were identified by RT-PCR at the CNM between 2005 and 2015 [16]. The genotype was studied in 591 of them and $543(91.9 \%)$ proved to belong to genotype G. The full $\mathrm{SH}$ gene of 357 of them was available for variant characterisation, from which 30 distinct haplotypes were identified (Figure 1).

278 samples (78\%) belonged to the same haplotype, displaying an identical $\mathrm{SH}$ sequence that was also identical to 346 of the 1,535 sequences from other countries that were available in GenBank. The WHO systematic name of the oldest sample was chosen to name the variant (MuVi/ Sheffield.GBR.1.05/[G]) [3].

Ability of non-coding regions to discriminate the SH subvariants of $\mathrm{MuVi}$ / Sheffield.

GBR.1.05/[G]

In 45 of the 46 selected samples, we successfully amplified and sequenced all three NCR fragments. The N-P containing fragment could not be amplified in one sample. Nested amplification was not required for the amplification of the $M-F$ region in 16 samples that showed a clear band after the first reaction. The length 
of the fragments used for phylogenetic analysis was $768 \mathrm{nt}(\mathrm{N}-\mathrm{P}), 758 \mathrm{nt}(\mathrm{M}-\mathrm{P})$ and $428 \mathrm{nt}(\mathrm{M}-\mathrm{F})$.

The sequence containing N-P showed 27 variable positions (one per $28.4 \mathrm{nt}, 96.4 \%$ conserved positions), while the sequence containing P-M had 11 (one per 68.8 nt, $98.5 \%$ conserved positions). Finally, the sequence containing $\mathrm{M}-\mathrm{F}$ proved to be the most variable with 23 variable positions (one per $18.6 \mathrm{nt}, 94.6 \%$ conserved positions). According to this, $\mathrm{M}-\mathrm{F}$ and $\mathrm{N}-\mathrm{P}$ regions both enabled discrimination of different groups in the phylogenetic tree, while well sustained clades were not observed for the P-M region (Figure 2). Consequently, we decided to proceed with the concatenation of the $\mathrm{M}-\mathrm{F}$ and N-P sequences, but not the P-M sequences.

As a consequence of the concatenation of the N-P and M-F NCR regions, it was possible to distinguish 24 haplotypes in selected 45 samples belonging to the MuVi/ Sheffield.GBR.1.05/[G] variant (Figure 3).

\section{Variant circulation}

The NCR variants detected in samples from 2005 to 2009 and those circulating from 2010 to 2015 formed different phylogenetic groups (Figure 3 ). The results were similar irrespective of whether they were based on the N-P or the M-F region (Figure 2, panels A and B). Two of the earliest strains (MuVs/Almería.ESP/20.05, MuVs/Cáceres.ESP/5.06) were the closest to the root of the tree. A well-supported subgroup of four sequences was observed within the 2010 to 2015 clade, obtained from patients involved in the same outbreak in the city of Murcia. Two exceptions to this temporal classification were observed: the MuVs/Zamora.ESP/23.11 strain isolated in 2011 grouped with those from 2005 to 2009. The opposite was the case for MuVs/Madrid.ESP/16.08 isolated in 2008, although this strain was located in a basal position in relation to the 2010 to 2015 clade.

\section{Discussion}

The main limitation of this study is that it was restricted to a single genotype (G). It would have been particularly interesting to reproduce the results on the genotype dominant before 2005 (genotype H), but we could not obtain enough material from sample collections.

Genotype $\mathrm{G}$ was the most frequently detected genotype in Spain during the period of the study, as well as in other western countries and Japan $[3,17,27]$. A particular variant of this genotype (MuVi/ Sheffield.GBR.1.05/ [G]) seemed to be dominant, not only in Spain but also in other countries, according to GenBank records. Such dominance of a single variant suggests continuous circulation of MuV [G], despite the sharp drop in incidence registered in Spain during the study period between the two epidemic waves [5]. In contrast, interruption of transmission of genotype $\mathrm{H}$ after a previous drop in incidence in 2004 was followed by the emergence of genotype $\mathrm{G}[5,15]$.
The WHO recommend genotyping MuV strains on the basis of the $\mathrm{SH}$ sequences in order to identify the patterns of circulation [3]. However, the $\mathrm{SH}$ region does not always provide enough resolution, and other genes such as $\mathrm{H}$ and $\mathrm{F}$ have been proposed as new markers to complement $\mathrm{SH}$ [28]. A recent study showed the NCR regions $N-P, P-M$ and $M-F$, in addition to the $S H$ gene, to be the most variable regions of the MuV genome [17]. Moreover, in the case of genotype G, the M-F NCR region seems to be more variable than the $\mathrm{SH}$ gene [17]. Our results are consistent with this conclusion. We found the M-F region to be the best molecular marker to complement the $\mathrm{SH}$ region in molecular epidemiology studies of the dominant genotype G. A short fragment of $428 \mathrm{nt}$ that includes this region proved to be easily amplified and sequenced by the proposed protocol, which makes it very suitable for the routine tasks carried out by most national reference laboratories into epidemiological surveillance and outbreak management of mumps in real time. However, according to a previous study [17], circumstances could be different for other genotypes, so this needs to be investigated further. In this context, the sequences containing NCR region $\mathrm{N}-\mathrm{P}$ proved to be a useful complement if additional discriminatory power is required.

The analysis of the sequences containing NCR variable regions $\mathrm{M}-\mathrm{F}$ and $\mathrm{N}-\mathrm{P}$ clearly revealed a strain replacement after the drop in incidence in 2009, which remained hidden after $\mathrm{SH}$ sequencing. The group of strains that subsequently emerged could be minor components of the viral populations circulating previously that would have been selected through a bottleneck mechanism because of decreased incidence. The evolutionary relationship between the two clades suggested by the phylogenetic tree topology, and the basal position of the strain MuVs/Madrid.ESP/16.08 in the 2010 to 2015 clade seem to support this hypothesis. Alternatively, they could have been imported from another geographical location after extinction of the previously circulating strains. Importation of other genotypes that cause sporadic cases and small outbreaks has been observed in the context of the circulation of genotype G [15]. Moreover, a genotype G strain (MuVs/Zamora.ESP/23.11) genetically related to those circulating from 2005 to 2009 was detected in 2011, suggesting either that it was imported from other parts of the world where these ancestral strains remained active after disappearing from Spain or that it continued circulating at low level. Additional data from the NCR regions of strains detected in other countries are needed to verify the importation hypothesis. Finally, the subgrouping of some sequences obtained from patients epidemiologically linked in the same outbreak suggests a potential use for these NCR regions in outbreak characterisation and discrimination of transmission chains. This merits further exploration.

We suggest that it would be worthwhile considering the use of these NCR regions, particularly M-F in genotype G, along with the SH gene, in the WHO's future 
recommendations for the epidemiological surveillance of MuV.

\section{Acknowledgements}

We would like to thank the Genomic Unit of the CNM-ISCIII for their technical assistance with sequencing. This work was supported by the "Fondo de Investigación Sanitaria" (FIS Pl12/02006) and the "Instituto de Salud Carlos III" (Pl15Clll/00023). Aurora Fernández García was funded by CIBER de Epidemiología y Salud Pública (CIBERESP), ISCIII.

\section{Conflict of interest}

None declared.

\section{Authors' contributions}

Ana María Gavilán: technical work, data analysis and writing as main author. Aurora Fernández-García: design for the study, data analysis, drafted and revised the manuscript. Angel Rueda: technical work and data analysis. Juan E Echevarría, design for the study, data analysis and writing as main author. Ana Castellanos: technical work. Josefa MasaCalles, Noemí López-Perea, María de Viarce Torres de Mier and Fernando de Ory reviewed and assisted in the editing of the final version of the manuscript.

\section{References}

1. Rubin SA. Sauder CJ, Carbone KM. Mumps virus. In: Knipe DM, Howley PM, editors. Fields Virology. Vol 2. 6th ed. Philadelphia: Lippincott, Williams and Wilkins, Wolters Kluwer Health; 2013. p.1025-41.

2. Richardson M, Elliman D, Maguire H, Simpson J, Nicoll A. Evidence base of incubation periods, periods of infectiousness and exclusion policies for the control of communicable diseases in schools and preschools. Pediatr Infect Dis J. 2001;20(4):380-91. https://doi.org/10.1097/00006454200104000-00004 PMID: 11332662

3. World Health Organization (WHO). Mumps virus nomenclature update: 2012. Wkly Epidemiol Rec. 2012;87(22):217-24. PMID: 24340404

4. Consejo Interterritorial del Sistema Nacional de Salud. Calendario común de vacunación infantil recomendado para 2016. [General childhood vaccination schedule recommended for 2016]. Madrid: Ministerio de Sanidad, Asuntos Sociales e Igualdad. [Accessed: May 2017]. Spanish. Available from: http://www.msssi.gob.es/ciudadanos/proteccionSalud/ vacunaciones/docs/CalendarioVacunacion2016.pdf

5. Red Nacional de Vigilancia Epidemiológica (RENAVE), Centro Nacional de Epidemiología. Situación de la Parotiditis en España, 1982-2016. [Situation of parotitis in Spain, 1982-2016]. Madrid: Instituto de Salud Carlos III; 2017. Spanish. Available from: http://www.isciii.es/ISCIII/es/contenidos/fd-servicioscientifico-tecnicos/fd-vigilancias-alertas/fd-enfermedades/ $\mathrm{fd}$-enfermedades-prevenibles-vacunacion/pdf_2017/Situacion Parotiditis_ES 1982_2016.pdf

6. Peltola H, Kulkarni PS, Kapre SV, Paunio M, Jadhav SS, Dhere RM. Mumps outbreaks in Canada and the United States: time for new thinking on mumps vaccines. Clin Infect Dis. 2007;45(4):459-66. https://doi.org/10.1086/520028 PMID: 17638194

7. Takla A, Wichmann O, Klinc C, Hautmann W, Rieck T, Koch J. Mumps epidemiology in Germany 2007-11. Euro Surveill. 2013;18(33):20557. https://doi.org/10.2807/1560-7917. ES2013.18.33.20557 PMID: 23968877

8. Patel LN, Arciuolo RJ, Fu J, Giancotti FR, Zucker JR, Rakeman JL, et al. Mumps outbreak among a highly vaccinated university community - New York City, January-April 2014. Clin Infect Dis. 2017;64(4):408-12. PMID: 27927872

9. Sane J, Gouma S, Koop-Mans M. de MH, Swaan C, van BR, et al. Epidemic of mumps among vaccinated persons, The Netherlands, 2009-2012. Emerg Infect Dis. 2014;20(4):643-8. PMID: 24655811
10. Eriksen J, Davidkin I, Kafatos G, Andrews N, Barbara C, Cohen D, et al. Seroepidemiology of mumps in Europe (1996-2008): why do outbreaks occur in highly vaccinated populations? Epidemiol Infect. 2013;141(3):651-66. https://doi.org/10.1017/ S0950268812001136 PMID: 22687578

11. Vygen S, Fischer A, Meurice L, Mounchetrou Njoya I, Gregoris $M$, Ndiaye $B$, et al. Waning immunity against mumps in vaccinated young adults, France 2013. Euro Surveill. 2016;21(10):30156. https://doi.org/10.2807/1560-7917. ES.2016.21.10.30156 PMID: 26987576

12. Nöjd J, Tecle T, Samuelsson A, Orvell C. Mumps virus neutralizing antibodies do not protect against reinfection with a heterologous mumps virus genotype. Vaccine. 2001;19(1314):1727-31. https://doi.org/10.1016/S0264-410X(00)00392-3 PMID: 11166897

13. Šantak M, Lang-Balija M, Ivancic-Jelecki J, Košutić-Gulija T, Ljubin-Sternak S, Forcic D. Antigenic differences between vaccine and circulating wild-type mumps viruses decreases neutralization capacity of vaccine-induced antibodies. Epidemiol Infect. 2013;141(6):1298-309. https://doi. org/10.1017/S0950268812001896 PMID: 22954346

14. Cilla G, Montes M, Zapico MS, Piñeiro L, Satrustegi M, Pérez-Yarza EG, et al. Genetic characterization of historical epidemic mumps viruses in northern Spain, 1987-1990. Infect Genet Evol. 2014;28:5-10. https://doi.org/10.1016/j. meegid.2014.08.025 PMID: 25176599

15. Echevarría JE, Castellanos A, Sanz JC, Pérez C, Palacios G, Martínez de Aragón MV, et al. Circulation of mumps virus genotypes in Spain from 1996 to 2007. J Clin Microbiol. 2010;48(4):1245-54. https://doi.org/10.1128/JCM.02386-09 PMID: 20107099

16. Royuela E, Castellanos A, Sánchez-Herrero C, Sanz JC, De Ory $F$, Echevarría JE. Mumps virus diagnosis and genotyping using a novel single RT-PCR. J Clin Virol. 2011;52(4):359-62. https:// doi.org/10.1016/j.jcv.2011.09.007 PMID: 21975077

17. Jin L, Örvell C, Myers R, Rota PA, Nakayama T, Forcic D, et al. Genomic diversity of mumps virus and global distribution of the 12 genotypes. Rev Med Virol. 2015;25(2):85-101. https:// doi.org/10.1002/rmv.1819 PMID: 25424978

18. Katoh K, Standley DM. MAFFT multiple sequence alignment software version 7: improvements in performance and usability. Mol Biol Evol. 2013;30(4):772-8o. https://doi. org/10.1093/molbev/mst010 PMID: 23329690

19. Hall TA. BioEdit: a user-friendly biological sequence alignment editor and analysis program for Windows 95/98/NT. Nucl Acids Symp. 1999;41:95-8.<jrn[REMOVED IF= FIELD]

20. Librado P, Rozas J. DnaSP v5: a software for comprehensive analysis of DNA polymorphism data. Bioinformatics. 2009;25(11):1451-2. https://doi.org/10.1093/bioinformatics/ btp187 PMID: 19346325

21. Stamatakis A. RAxML version 8: a tool for phylogenetic analysis and post-analysis of large phylogenies. Bioinformatics. 2014;30(9):1312-3. https://doi.org/10.1093/ bioinformatics/btu033 PMID: 24451623

22. Guindon S, Dufayard JF, Lefort V, Anisimova M, Hordijk W, Gascuel 0 . New algorithms and methods to estimate maximum-likelihood phylogenies: assessing the performance of PhyML 3.0. Syst Biol. 2010;59(3):307-21. https://doi. org/10.1093/sysbio/syq010 PMID: 20525638

23. Santorum JM, Darriba D, Taboada GL, Posada D. jmodeltest. org: selection of nucleotide substitution models on the cloud. Bioinformatics. 2014;30(9):1310-1. https://doi.org/10.1093/ bioinformatics/btuo32 PMID: 24451621

24. Gouy M, Guindon S, Gascuel O. SeaView version 4: A multiplatform graphical user interface for sequence alignment and phylogenetic tree building. Mol Biol Evol. 2010;27(2):2214. https://doi.org/10.1093/molbev/msp259 PMID: 19854763

25. Lanfear R, Calcott B, Ho SYW, Guindon S. Partitionfinder: combined selection of partitioning schemes and substitution models for phylogenetic analyses. Mol Biol Evol. 2012;29(6):1695-701. https://doi.org/10.1093/molbev/msso20 PMID: 22319168

26. Tamura K, Stecher G, Peterson D, Filipski A, Kumar S. MEGA6: Molecular Evolutionary Genetics Analysis version 6.0. Mol Biol Evol. 2013;30(12):2725-9. https://doi.org/10.1093/molbev/ mst197 PMID: 24132122

27. Aoki Y, Matoba Y, Tanaka S, Yahagi K, Itagaki T, Katsushima $\mathrm{F}$, et al. Chronological changes of mumps virus genotypes in Japan between 1999-2013. Infect Dis (Lond). 2016;48(7):5249. https://doi.org/10.3109/23744235.2016.1163730 PMID: 27206988

28. Gouma S, Cremer J, Parkkali S, Veldhuijzen I, van Binnendijk RS, Koopmans MPG. Mumps virus F gene and HN gene sequencing as a molecular tool to study mumps virus transmission. Infect Genet Evol. 2016;45:145-50. https://doi. org/10.1016/j.meegid.2016.08.033 PMID: 27590714 


\section{License and copyright}

This is an open-access article distributed under the terms of the Creative Commons Attribution (CC BY 4.0) Licence. You may share and adapt the material, but must give appropriate credit to the source, provide a link to the licence, and indicate if changes were made.

This article is copyright of the authors, 2018. 